\title{
Bandpass Effects in Time-Resolved Diffuse Spectroscopy
}

\author{
A. FARINA,* A. BASSI, A. PIFFERI, P. TARONI, D. COMELLI, L. SPINELLI, and R. CUBEDDU \\ IIT, CNR-INFM and CNR-IFN, Dipartimento di Fisica, Politecnico di Milano, Piazza Leonardo da Vinci 32, 20133 Milano, Italy
}

\begin{abstract}
This paper discusses the spectral distortions occurring when time-resolved spectroscopy of diffusive media is performed illuminating with a wide bandpass. It is shown that the spectral region within the bandpass that exhibits the lowest absorption will dominate the resulting time-resolved curve, leading to significant underestimations of absorption as well as distortions in the spectral shape (including shifts in peak positions). Due to the nonlinear behavior of absorption, this effect becomes even more pronounced when including longer and longer photon path lengths. First, a theoretical treatment of the problem is given, and then the distortion is described by time-resolved reflectance simulations and experimental measurements of lipid and water samples. A spectrally constrained data analysis is proposed that takes into account the spectrum of the light injected into the sample, used to overcome the distortion and improve the accuracy of the estimation of chromophore concentrations from absorption spectra. Measurements on a lipid sample show a reduction of the error from $30 \%$ to $6 \%$.

Index Headings: Time-resolved spectroscopy; Turbid media; Diffusive media; Supercontinuum; Spectral distortion.
\end{abstract}

\section{INTRODUCTION}

It is well known that any spectroscopy system introduces distortions in the measured spectra due to the non-ideal instrumental spectral response function. In particular, the effects of the finite slit width of a spectrophotometer have been widely discussed, ${ }^{1-5}$ showing that the recovered spectra can be significantly distorted if the spectral bandpass of the instrument is comparable with the absorption linewidth. The bandpass is given by the combination of the spectral line of the source and the spectral response of the detector. Furthermore, Brodersen ${ }^{2}$ discussed the effect of a sample with large optical density on the calculation of the extinction coefficient, showing that distortions increase with the sample optical density and consequently with the lengthening of the photon path inside the medium.

The path of photons in a diffusive medium is much longer than in a transparent medium. In highly diffusive media, such as biological tissues, the probability that photons will undergo scattering events is higher than the probability that photons will undergo absorption events. This causes their trajectories to be modified, resulting in a longer overall path. For instance, photon mean path length after transmission through a $4 \mathrm{~cm}$ thick breast tissue can be approximately $100 \mathrm{~cm}$. Therefore, it is expected that the spectral distortions will be further amplified in highly diffusive media. In the study of light propagation inside highly diffusive media ("photon migration"), the photon path distribution can be taken into account by means of a proper theory. ${ }^{6}$ Photon migration has become a fundamental tool for the characterization of biological tissues and has been applied to noninvasive breast cancer investigation, ${ }^{7}$ to human brain functional imaging, ${ }^{8}$ and to molecular imaging. ${ }^{9}$ Concerning industrial applications, photon migration has been

Received 14 August 2008; accepted 21 October 2008. * Author to whom correspondence should be sent. E-mail: andrea.
farina@polimi.it. applied to the estimation of drug concentrations in pharmaceutical tablets ${ }^{10}$ and to the characterization of wood $^{11}$ and fruit. ${ }^{12}$ In particular, biological tissues present low absorption in the range from 600 to $1100 \mathrm{~nm} \cdot{ }^{13}$ In this "therapeutic window" it is possible to quantify, by means of diffuse absorption spectroscopy, the concentrations of the main biological chromophores: water, lipid, collagen, and oxy- and deoxy-hemoglobin.

Rapid technological development in this area has made available new compact laser sources suitable for photon migration studies. In particular, diode lasers and high-power fiber lasers pumping photonic crystal fibers and generating supercontinuum radiation have been developed. ${ }^{14,15}$ Instruments based on these technologies have the great advantage of being compact and suitable for clinical applications but have the disadvantage of presenting a non-ideal spectral bandpass. It is therefore important to understand the effects induced by a broad bandpass on the retrieved absorption spectrum of highly diffusive media. Several approaches to diffuse spectroscopy have been proposed: time resolved (TR), ${ }^{16-19}$ steady state (SS), ${ }^{20}$ and frequency domain (FD) combined with SS. ${ }^{21}$ Spectral distortions are common for different approaches because the methods are governed by the long photon path; however, this long path length can be significantly reduced depending on the bandpass of the instruments. In this work we systematically analyze the effects of a broad bandpass on the estimation of absorption spectra in time-resolved diffuse spectroscopy. We first give a theoretical interpretation of the effect, then simulations of near-infrared absorption spectra of water and lipid are shown, and then we report on an experimental investigation. Finally, a method to compensate the bandpass effect on the retrieved absorption spectra is proposed and validated experimentally.

\section{THEORY}

This section presents an analytical description of the effect of the bandpass on time-resolved spectroscopy of diffusive media. In particular, it is shown how the photon fluence rate and the estimation of the absorption spectrum depend on the bandpass of the system and on the photon time of flight (TOF).

Light propagation in diffusive media can be described by the radiative transport equation: ${ }^{6}$

$$
\begin{aligned}
\frac{1}{v} \frac{\partial L(\boldsymbol{r}, \Omega, t)}{\partial t}= & -\Omega \cdot \nabla L(\boldsymbol{r}, \Omega, t)-\left(\mu_{\mathrm{a}}+\mu_{\mathrm{s}}\right) L(\boldsymbol{r}, \Omega, t) \\
& +\mu_{\mathrm{s}} \int_{4 \pi} p\left(\Omega, \Omega^{\prime}\right) L\left(\boldsymbol{r}, \Omega^{\prime}, t\right) \mathrm{d} \Omega^{\prime}+S(\boldsymbol{r}, \Omega, t)
\end{aligned}
$$

where $L(\boldsymbol{r}, \Omega, t)$ is the radiance in direction $\Omega, v$ is the light speed inside the medium, $\mu_{\mathrm{a}}$ and $\mu_{\mathrm{s}}$ are, respectively, the absorption and scattering coefficient, $p\left(\Omega, \Omega^{\prime}\right)$ is the scattering phase function, and $S(\boldsymbol{r}, \Omega, t)$ is the source term. ${ }^{22}$ If the source term is a Dirac delta function in time and monochromatic, Eq. 1 
has the following property: if $L_{0}(\boldsymbol{r}, \Omega, t)$ is the solution for a nonabsorbing medium, $L(\boldsymbol{r}, \Omega, t)=L_{0}(\boldsymbol{r}, \Omega, t) \exp \left(-\mu_{\mathrm{a}} v t\right)$ is the solution to Eq. 1 when $\mu_{\mathrm{a}}$ is the spatially independent absorption coefficient. ${ }^{22}$ Consequently the detected photon fluence rate at any position $\boldsymbol{r}_{\mathrm{D}}$ can be written as:

$$
I\left(\boldsymbol{r}_{\mathrm{D}}, t\right)=I_{0}\left(\boldsymbol{r}_{\mathrm{D}}, t, \mu_{\mathrm{s}}\right) \exp \left(-\mu_{\mathrm{a}} v t\right)
$$

where $I_{0}\left(\boldsymbol{r}_{\mathrm{D}}, t, \mu_{\mathrm{s}}\right)$ is the detected power in the non-absorbing medium and takes into account the geometry, the boundary conditions, and the scattering properties of the medium. The absorption coefficient is therefore proportional to the slope of the logarithm of the ratio between the detected fluence rate in the absorbing medium and a non-absorbing medium that has the same scattering.

If the light source is not monochromatic, the photon fluence rate detected at the position $\boldsymbol{r}_{\mathrm{D}}$ is given by the following integral:

$$
I^{*}\left(\lambda ; \boldsymbol{r}_{\mathrm{D}}, t\right)=\int g\left(\lambda ; \lambda^{\prime}\right) I\left[\boldsymbol{r}_{\mathrm{D}}, t ; \mu_{\mathrm{a}}\left(\lambda^{\prime}\right), \mu_{\mathrm{s}}\left(\lambda^{\prime}\right)\right] \mathrm{d} \lambda^{\prime}
$$

where $g\left(\lambda ; \lambda^{\prime}\right)$ is the normalized spectrum of the source at the nominal wavelength $\lambda$. Equation 3 represents a weighted average of the time-resolved intensity profiles that fall within the spectral band of the source, and the measurement of the profile at $\lambda$ is thus distorted by cross-talk from neighboring wavelengths. It can be observed that $\lambda$ can be either the wavelength set in the spectrometer or the nominal emission wavelength of a laser, i.e., when a diode laser is used as a light source. Assuming a constant scattering over the bandpass of the system, Eq. 3 reduces to

$$
I^{*}\left(\lambda ; \boldsymbol{r}_{\mathrm{D}}, t\right)=I_{0}\left(\boldsymbol{r}_{\mathrm{D}}, t, \mu_{\mathrm{s}}\right) \int g\left(\lambda ; \lambda^{\prime}\right) \exp \left[-\mu_{\mathrm{a}}\left(\lambda^{\prime}\right) v t\right] \mathrm{d} \lambda^{\prime}
$$

The absorption coefficient can be estimated by taking the first temporal derivative of the logarithm of the ratio $I^{*} / I_{0}$ :

$$
\begin{aligned}
\mu_{\mathrm{a}}^{*}(\lambda ; t) & =-\frac{1}{v} \frac{\mathrm{d}}{\mathrm{d} t} \ln \left[\frac{I^{*}\left(\lambda ; \boldsymbol{r}_{\mathrm{D}}, t\right)}{I_{0}\left(\boldsymbol{r}_{\mathrm{D}}, t, \mu_{s}\right)}\right] \\
& =\frac{\int g\left(\lambda ; \lambda^{\prime}\right) \exp \left[-\mu_{\mathrm{a}}\left(\lambda^{\prime}\right) v t\right] \mu_{\mathrm{a}}\left(\lambda^{\prime}\right) \mathrm{d} \lambda^{\prime}}{\int g\left(\lambda ; \lambda^{\prime}\right) \exp \left[-\mu_{\mathrm{a}}\left(\lambda^{\prime}\right) v t\right] \mathrm{d} \lambda^{\prime}}
\end{aligned}
$$

It is worth noting that the estimation of the absorption coefficient $\mu_{\mathrm{a}}^{*}$ will depend on the photon TOF used, but it does not depend on the detector position $\boldsymbol{r}_{\mathrm{D}}$ or on the scattering coefficient $\mu_{\mathrm{s}}$. It is important to point out that in a real case the fitting procedure is performed over a temporal range and that the function $I_{0}$ is not measured. It can be observed that if $\mathrm{g}(\lambda$; $\lambda^{\prime}$ ) tends to a Dirac delta function, $\mu_{\mathrm{a}}^{*}$ tends to the real absorption coefficient $\mu_{\mathrm{a}}(\lambda)$.

Equation 5 can be reduced to the following:

$$
\mu_{\mathrm{a}}^{*}(\lambda ; t)=\int w\left(\lambda ; \lambda^{\prime}, t\right) \mu_{\mathrm{a}}\left(\lambda^{\prime}\right) \mathrm{d} \lambda^{\prime}
$$

where $w\left(\lambda ; \lambda^{\prime}, t\right)$ is a weight function equal to

$$
w\left(\lambda ; \lambda^{\prime}, t\right)=\frac{g\left(\lambda ; \lambda^{\prime}\right) \exp \left[-\mu_{\mathrm{a}}\left(\lambda^{\prime}\right) v t\right]}{\int g\left(\lambda ; \lambda^{\prime}\right) \exp \left[-\mu_{\mathrm{a}}\left(\lambda^{\prime}\right) v t\right] \mathrm{d} \lambda^{\prime}}
$$

Equation 6 indicates that the estimated absorption coefficient at the wavelength $\lambda$ is a weighted average of the true absorption spectrum of the medium (the part that falls within the bandpass) and will depend on the photon TOF used. The weight function $w\left(\lambda ; \lambda^{\prime}, \mathrm{t}\right)$ can be factorized into two terms, $g\left(\lambda ; \lambda^{\prime}\right)$ and $\exp \left[-\mu_{\mathrm{a}}\left(\lambda^{\prime}\right) v t\right]$. The first one takes into account the spectral response function of the system and the second one the nonlinear attenuation due to absorption.

As often reported in the literature, ${ }^{1-5} \mu_{\mathrm{a}}\left(\lambda^{\prime}\right)$ and $g\left(\lambda ; \lambda^{\prime}\right)$ are assumed to be Gaussian functions, in order to better understand the behavior of the photon fluence rate $I^{*}\left(\lambda ; \boldsymbol{r}_{\mathrm{D}}, t\right)$ and the weight function $w\left(\lambda ; \lambda^{\prime}, t\right)$. The absorption and spectral response functions are

$$
\mu_{\mathrm{a}}\left(\lambda^{\prime}\right)=\mu_{\mathrm{a} 0} \exp \left[-\frac{4\left(\lambda^{\prime}-\lambda_{0}\right)^{2}}{W_{\mathrm{a}}^{2}} \ln 2\right]
$$

and

$$
g\left(\lambda ; \lambda^{\prime}\right)=\frac{2}{W_{\mathrm{g}}}\left(\frac{\ln 2}{\pi}\right)^{1 / 2} \exp \left[-\frac{4\left(\lambda^{\prime}-\lambda\right)^{2}}{W_{\mathrm{g}}^{2}} \ln 2\right]
$$

where $\mu_{\mathrm{a} 0}$ is the peak absorption coefficient, $\lambda_{0}$ is the absorption central wavelength, and $W_{\mathrm{a}}$ and $W_{\mathrm{g}}$ are the width (full width at half-maximum, FWHM) of the absorption line and the system bandpass (FWHM), respecitvely. By inserting Eqs. 8 and 9 into Eqs. 4 and 7 it is possible to obtain normalized plots of $I^{*}(\lambda) / I_{0}$ as functions of the ratio $S$ between $W_{\mathrm{g}}$ and $W_{\mathrm{a}}$. Figure 1 shows the ratio $I^{*}(\lambda) / I_{0}$ versus photon TOF, calculated on the peak and on the inflection point of the Gaussian absorption spectrum, for different bandpasses. This shows how the time-resolved curves are modified by the wide bandpass of the system. It can be observed that for $S=W_{\mathrm{g}} / W_{\mathrm{a}}$ $\neq 0$ the slope drops to lower values at increasing TOFs; this reduction is emphasized by the increase of $S=W_{\mathrm{g}} / W_{\mathrm{a}}$.

The behavior shown in Fig. 1 may be explained by taking into account the fact that the ratio $I^{*}(\lambda) / I_{0}$ is a weighted sum of Lambert-Beer terms over wavelength and spectral regions with low absorption give a strong contribution to the fluence. Due to the nonlinearity of attenuation with respect to path length, this effect is emphasized at longer photon TOFs. Furthermore it can be observed that this trend is more pronounced on the inflection point of the absorption spectrum, where the slope of the spectrum is maximum. In fact, in the region of overlap between $\mu_{\mathrm{a}}\left(\lambda^{\prime}\right)$ and $g\left(\lambda ; \lambda^{\prime}\right)$ the absorption spans a wider range.

The weight function $w\left(\lambda ; \lambda^{\prime}, t\right)$ depends on the photon TOFs and the bandpass. In Fig. 2 it can be seen that for a narrow bandpass the weight function is concentrated in the neighborhood of the reference wavelength $\lambda$ with a small dependence on TOF; consequently, it will not appreciably affect the estimated absorption. For a wider bandpass the weight function considerably changes with TOF, enhancing the cross-talk with regions of the spectrum with low absorption. This will cause an increasing underestimation of the absorption with TOF. The wider the bandpass is, the earlier the cross-talk effect will be important. To better understand how these distortions affect the absorption spectrum, $\mu_{\mathrm{a}}^{*}(\lambda ; t)$ (Eq. 6) has been calculated for three TOFs (Fig. 3). The distortion introduced by the bandpass is present at all TOFs and causes an amplitude reduction and a linewidth change of the estimated absorption spectrum. This behavior is summarized in Fig. 4, where the maximum amplitude and the linewidth are plotted as a function of TOF 

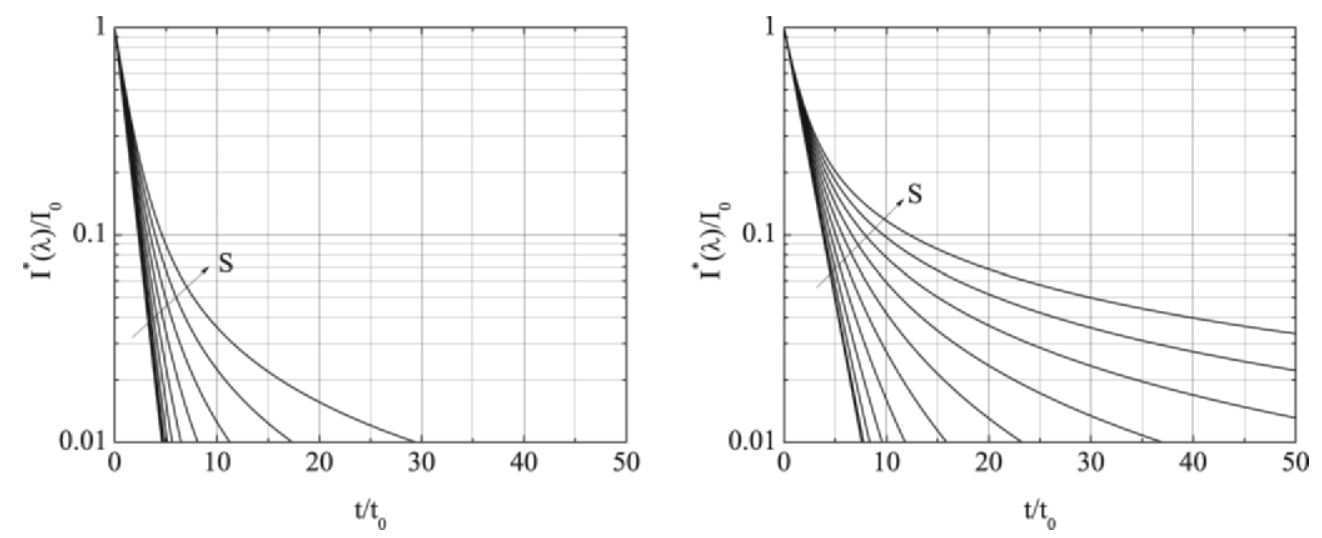

FIG. 1. Intensity of signal normalized to the intensity of a non-absorbing medium $\left(I^{*}(\lambda) / I_{0}\right)$ versus normalized photon TOF, (left) on the peak and (right) on the inflection point of the absorption spectrum. $t_{0}=1 /\left(\mu_{\mathrm{a} 0} v\right) . S=W_{\mathrm{g}} / W_{\mathrm{a}}=[0,1]$ is the ratio between the absorption bandwidth (FWHM) and the bandpass of the system (FWHM). The asymptotic slope changes due to the cross-talk between high and low absorption regions of the spectrum. The effect is enhanced on the inflection point.

for different bandpasses. Figure 4 (left panel) confirms that the contribution of the low absorption regions of the spectrum is enhanced if the bandpass is increased; therefore, the absorption peak estimation presents a nearly flat behavior with respect to TOF after a fast decrease. This trend is similar at all wavelengths: the farther from the peak the wavelength is, the steeper is the absorption amplitude at early TOFs. This explains the trend of absorption width shown in Fig. 4 (right panel): the curve presents a minimum that is given by the different behavior of the estimated absorption at the peak and the offpeak wavelengths.

It can be also observed that the peak position of the true spectrum is still preserved; in fact, if both $g\left(\lambda ; \lambda^{\prime}\right)$ and $\mu_{\mathrm{a}}^{*}(\lambda ; t)$ are symmetrical, $\mu_{\mathrm{a}}^{*}(\lambda ; t)$ is symmetrical too (Eq. 5). If a nonsymmetrical absorption line is considered, another distortion is introduced. Figure 5 shows the calculated absorption spectra at three different TOFs. The spectrum plotted in Fig. 5 is described by the following equation:

$$
\begin{aligned}
\mu_{\mathrm{a}}= & \mu_{\mathrm{a} 0}+A \frac{1}{1+\exp \left[-\left(\lambda^{\prime}-\lambda_{0}+w_{1} / 2\right) / w_{2}\right]} \\
& \left\{1-\frac{1}{1+\exp \left[-\left(\lambda^{\prime}-\lambda_{0}+w_{1} / 2\right) / w_{3}\right]}\right\}
\end{aligned}
$$

where $\mu_{\mathrm{a} 0}, A, w_{1}, w_{2}, w_{3}$, and $\lambda_{0}$ are arbitrary parameters.

It can be observed that, besides the already discussed
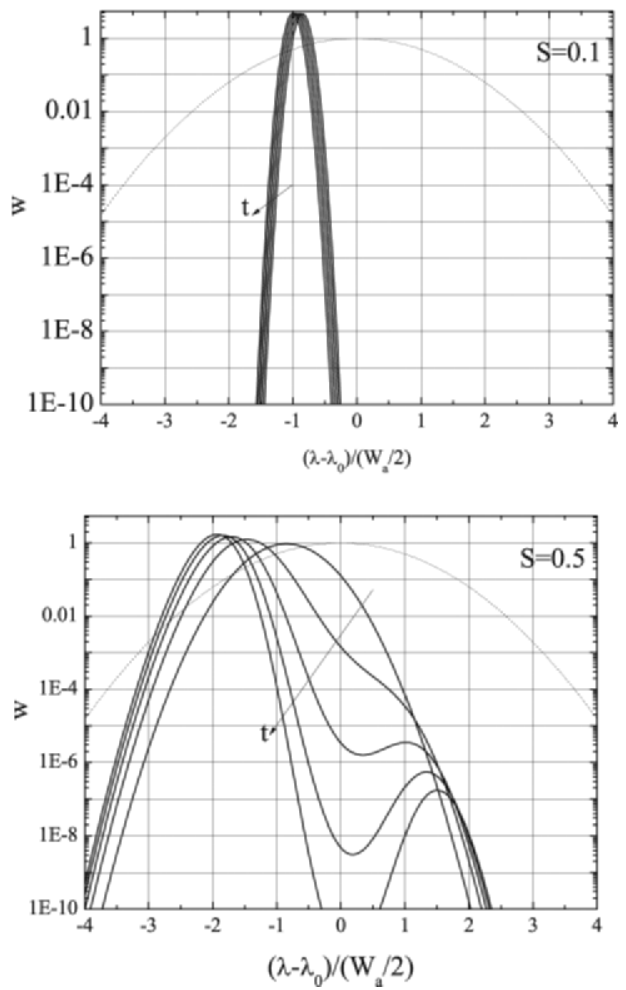

FIG. 2. Weight function versus $\lambda$ (solid line) plotted on (left column) the peak and (right column) the inflection point of the absorption spectrum for $t=\left[0,30 t_{0}\right]$,

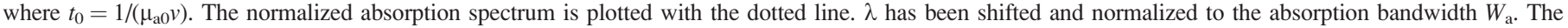
weight function depends on the photon TOF weakly for a small value of $S=W_{\mathrm{g}} / W_{\mathrm{a}}$ and strongly for a high value of $S$. 
distortion, a peak shift to longer wavelengths is present, and this distortion is increased with the photon TOF. In Fig. 6 the peak shift is plotted as a function of TOF for different bandpasses, indicating that the increasing width of the bandpass enhances the peak shift.

This peak shift can be analytically explained by taking the first derivate of the logarithm of Eq. 6 with respect to $\lambda$ :

$$
\begin{aligned}
\frac{\partial \ln \mu_{\mathrm{a}}^{*}}{\partial \lambda}= & \frac{\int g^{\prime}\left(\lambda ; \lambda^{\prime}\right) \exp \left[-\mu_{\mathrm{a}}\left(\lambda^{\prime}\right) v t\right] \mathrm{d} \lambda^{\prime}}{\int g^{\prime}\left(\lambda ; \lambda^{\prime}\right) \exp \left[-\mu_{\mathrm{a}}\left(\lambda^{\prime}\right) v t\right] \mathrm{d} \lambda^{\prime}} \\
& -\frac{\int g^{\prime}\left(\lambda ; \lambda^{\prime}\right) \exp \left[-\mu_{\mathrm{a}}\left(\lambda^{\prime}\right) v t\right] \mu_{\mathrm{a}}\left(\lambda^{\prime}\right) \mathrm{d} \lambda^{\prime}}{\int g^{\prime}\left(\lambda ; \lambda^{\prime}\right) \exp \left[-\mu_{\mathrm{a}}\left(\lambda^{\prime}\right) v t\right] \mu_{\mathrm{a}}\left(\lambda^{\prime}\right) \mathrm{d} \lambda^{\prime}}
\end{aligned}
$$

If both $g\left(\lambda ; \lambda^{\prime}\right)$ and $\mu_{\mathrm{a}}\left(\lambda^{\prime}\right)$ are symmetrical functions, the right side of Eq. 11 calculated on the absorption peak is zero. This means that the peak position is preserved. If the absorption is not symmetrical, the term on the right hand side of Eq. 11 calculated on the absorption peak is different from zero. In particular, if the rising edge of $\mu_{\mathrm{a}}\left(\lambda^{\prime}\right)$ is steeper than the falling edge, the right side of Eq. 11 calculated on the peak wavelength results in a solution higher than zero. Conversely, if the rising edge of $\mu_{\mathrm{a}}\left(\lambda^{\prime}\right)$ is less steep than the falling edge, the term on the right side of Eq. 11 calculated on the peak wavelength is lower than zero. In these two cases a shift to longer and shorter wavelengths of the absorption peak is present, respectively.

\section{SIMULATIONS}

Photon migration is a powerful tool for the noninvasive characterization of diffusive media such as biological tissues. A typical experimental configuration consists of injection and collection fibers, placed at some distance (inter-fiber distance) on the same surface of the sample. In time-resolved measurements a short pulse is injected in the sample by means of the first fiber and the power collected by the second fiber is coupled to a fast detector. Typically the collected photon fluence rate is fitted to an analytical solution of the diffusion approximation of Eq. 1 in order to retrieve the absorption and the reduced scattering coefficients. ${ }^{22}$ Therefore, absorption and scattering spectra can be obtained by performing measurements at different wavelengths. In particular water, lipid, and hemoglobin are the main constituents of biological tissues such as breast tissue, muscle tissue, and brain tissue. Tissue composition and related important diagnostic information can be derived from the absorption spectra of tissues. Thus, it is important to show the distortions induced by the instrumental bandpass on the retrieved absorption spectra of these chromophores. In the following we will focus on the water and lipid absorption spectra because, in addition to the distortion presented in the Theory section, they also show the absorption peak shift.

To investigate the bandpass effect on water and lipid spectra, theoretical reflectance curves in the diffusion approximation were first generated in the range from 600 to $1100 \mathrm{~nm}$. Then, assuming a Gaussian spectral response of the instrument, the weighted superposition of Eq. 3 was obtained for every wavelength $\lambda$. This procedure was used to generate reflectance curves at inter-fiber distances of $0.5,1,2$, and $4 \mathrm{~cm}$, at $\mu_{\mathrm{s}}^{\prime}$
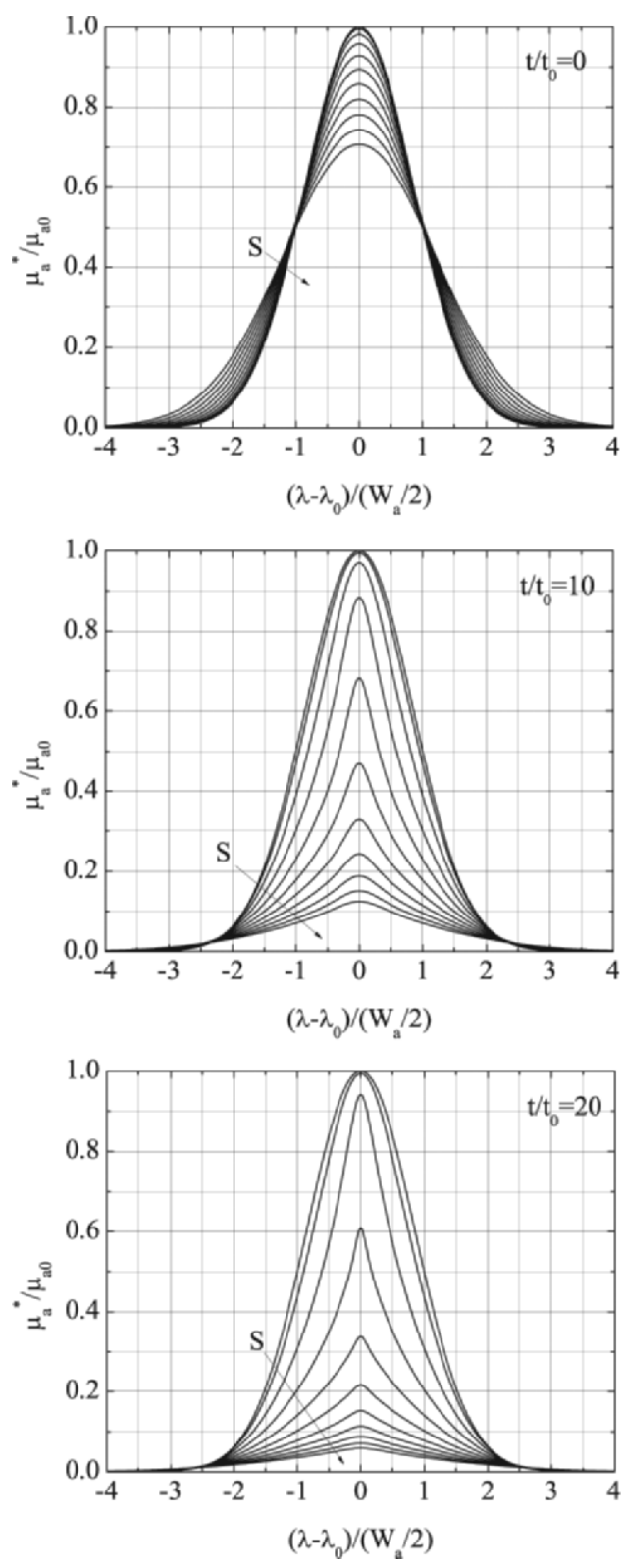

FIG. 3. Calculated absorption spectrum at three different TOFs and bandpasses when the absorption line is a Gaussian function. $S=W_{\mathrm{g}} / W_{\mathrm{a}}=$ $[0,1]$ and $t_{0}=1 /\left(\mu_{\mathrm{a} 0} v\right)$. The distortion due to the increase of $S$ is enhanced at increasing photon TOFs.

values of 5,10 , and $15 \mathrm{~cm}^{-1}$ and with bandpasses ranging from 0 to $30 \mathrm{~nm}$ (FWHM). Inter-fiber distance and reduced scattering values are typical for in vivo measurements of biological tissues. The generated data set was then fitted by the Levenberg-Marquardt least squares algorithm ${ }^{23}$ to the diffusive time-resolved reflectance with extrapolated boundary conditions model for a semi-infinite medium. ${ }^{22,24}$ The fitting range included all points with a number of counts higher than $80 \%$ of the peak value on the rising edge of the curve and $1 \%$ on the tail.

In Figs. 7 and 8 simulation results and percentage errors are shown for the retrieved water and lipid spectra, respectively. Results are grouped by $\rho^{2} \mu^{\prime}{ }_{\text {s }}$ product, where $\rho$ is the inter-fiber distance, because time-resolved reflectance curves with the same $\rho^{2} \mu^{\prime}$ s product are very similar. ${ }^{25}$ Figures 7 and 8 show the 

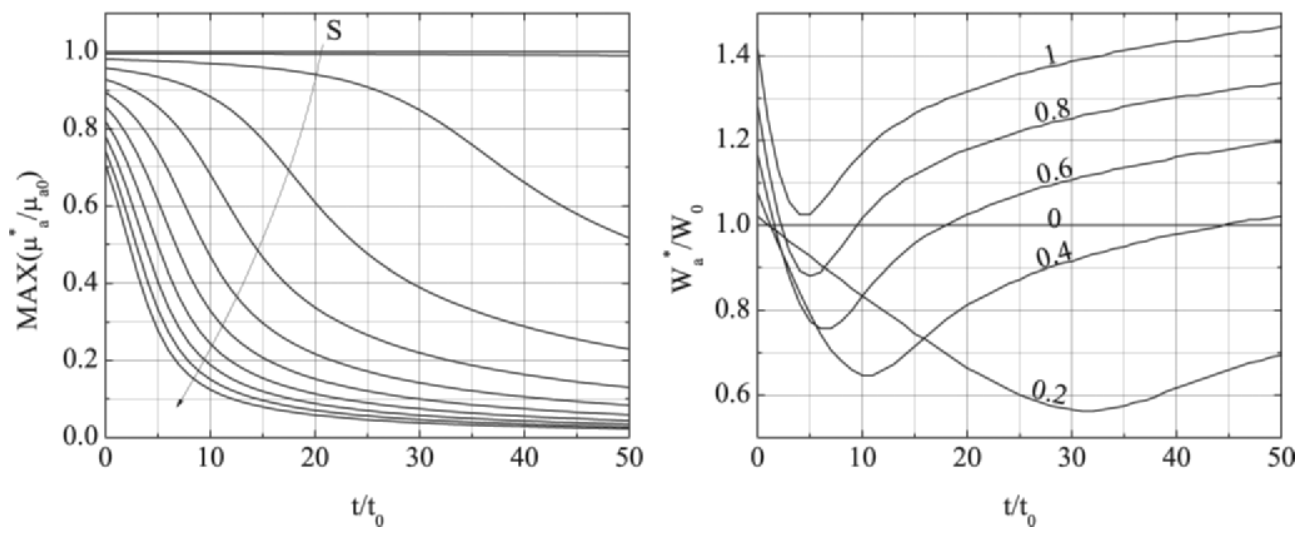

FIG. 4. (Left) Absorption peak amplitude versus normalized photon TOF for $S=W_{\mathrm{g}} / W_{\mathrm{a}}$ ranging from 0 to $1 . t_{0}=1 /\left(\mu_{\mathrm{a} 0} v\right)$. (Right) Ratio between calculated ( $\left.W_{\mathrm{a}}^{*}\right)$ and real $\left(W_{0}\right)$ absorption width (FWHM) for different $S=W_{\mathrm{g}} / W_{\mathrm{a}}$ (as indicated over the curve). The trends shown are due to the fact that the cross-talk between low and high absorption regions of the spectrum is not linear, on the wavelength $\lambda$, and on the photon TOF used.
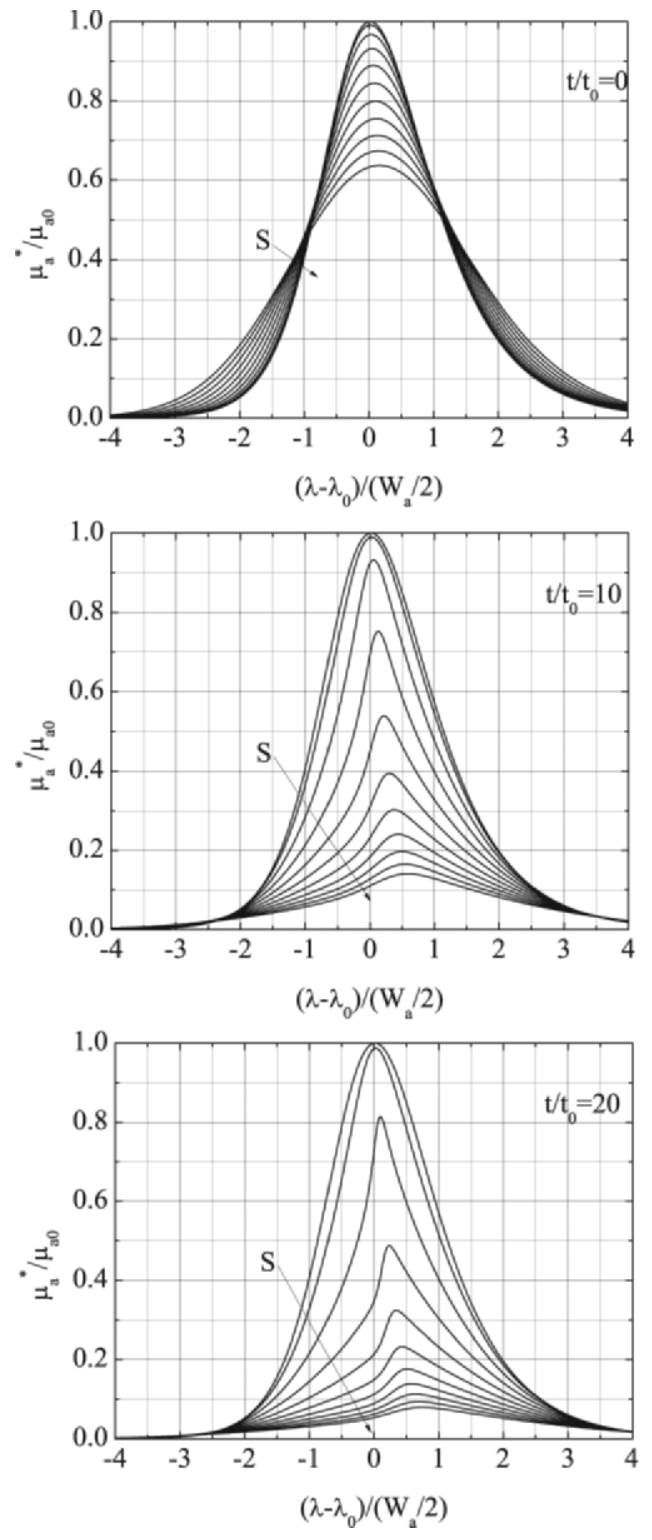

Fig. 5. Calculated absorption spectrum at three different TOFs and bandpasses when the absorption line is nonsymmetrical. $S=W_{\mathrm{g}} / W_{\mathrm{a}}=[0,1]$ and $t_{0}=1 /\left(\mu_{\mathrm{a} 0} v\right)$. Besides the distortion discussed for a symmetrical absorption line, an absorption peak shift is present. distortions of the spectra: it can be observed that the absorption peak shifts to longer wavelengths for the water and to shorter wavelengths for the lipid. Significant differences can also be observed at wider bandpasses: the error shown in Figs. 7 and 8 can reach $60 \%$ on the maximum slope of the absorption line. It can also be noted that there is an increasing distortion with the $\rho^{2} \mu_{\text {s }}^{\prime}$ product given by the fact that the time-resolved reflectance curve is delayed and broadened at increasing $\rho^{2} \mu^{\prime}$. Since the fitting range is referred to the peak of the temporal curve, by increasing $\rho^{2} \mu^{\prime}$ s the range shifts toward longer TOFs. The trend of the distortion with $\rho^{2} \mu^{\prime}$ s demonstrates that if photons running a long path are used to evaluate the absorption coefficient, then the distortion on the estimation of the absorption coefficient is enhanced.

\section{EXPERIMENTAL WORK}

In order to experimentally demonstrate the effect of the instrumental bandpass on the recovery of the absorption coefficient, time-resolved measurements are performed using two spectroscopic systems. The first one (TiS) is based on a tunable mode-locked Ti:sapphire laser, ${ }^{19}$ and the second one (SC) is based on a high-power fiber laser generating supercontinuum radiation in the range $465-1750 \mathrm{~nm} .{ }^{26} \mathrm{TiS}$ presents a spectral response function with a width (FWHM) $<1 \mathrm{~nm}$ for each wavelength in the range 700-1100 nm, allowing

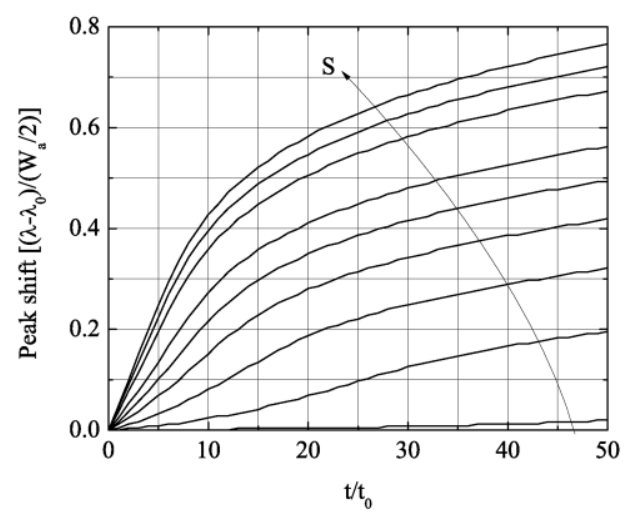

FIG. 6. Estimated normalized peak wavelength versus normalized TOF $\left(t_{0}=\right.$ $\left.1 /\left(\mu_{\mathrm{a} 0} v\right)\right)$ for $S=W_{\mathrm{g}} / W_{\mathrm{a}}$ ranging from 0 to 1 . The shift depends on the TOF used. 

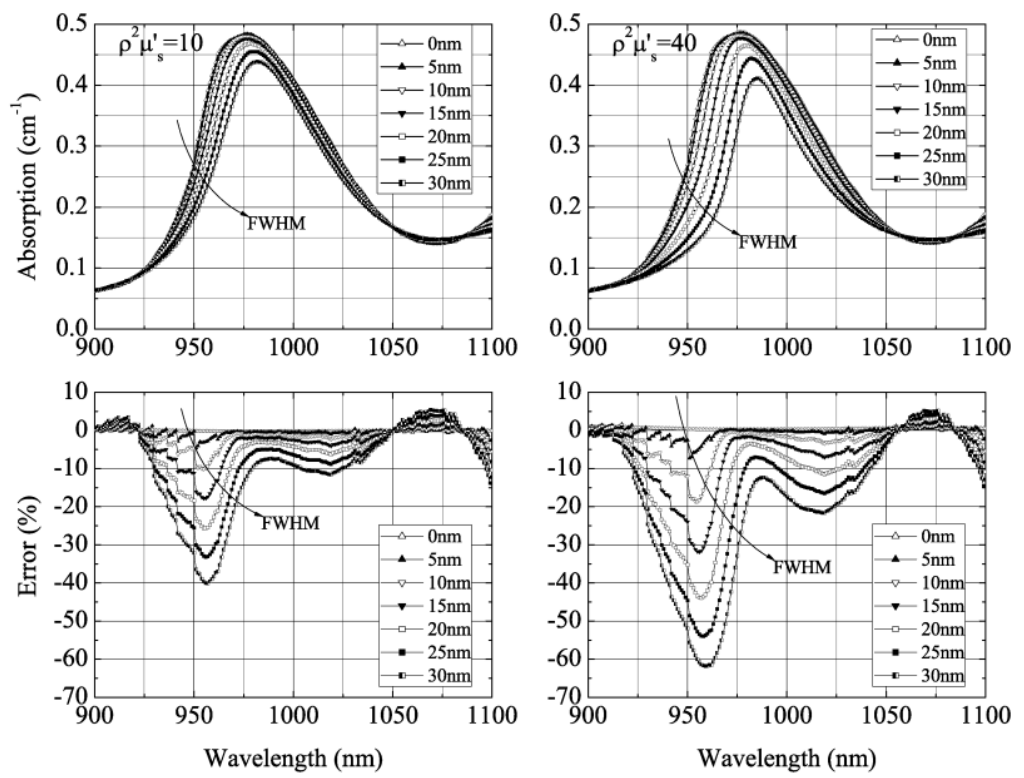

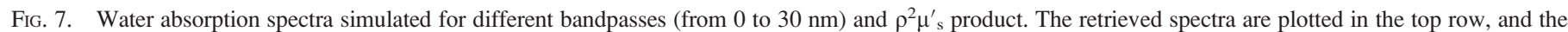
percentage errors are plotted in the bottom row. It can be seen that the absorption peak shifts toward higher wavelengths for increasing bandpasses.

experimental work that is equivalent to having an ideal spectral response. In the SC method, light is dispersed by a prism and consequently focused by an achromatic lens to an optical fiber. The bandpass of this system (SC) was changed in order to obtain two different configurations (called SC1 and SC2). Figure 9 shows the bandpass in the two cases. For both systems the detected light was coupled to a cooled micro-channel plate photomultiplier tube (1564U, Hamamatsu, Japan) with a S1 surface that offers a temporal resolution of about $50 \mathrm{ps}$ and a quantum efficiency less than $0.1 \%$ at $1100 \mathrm{~nm}$. The spectrum of the light entering the sample is measured online by an optical multi-channel analyzer (OMA) with a stability better than $10 \%$ over the measurement time $(\sim 1 \mathrm{~s})$. A schematic of the system is depicted in Fig. 10.

Two phantoms were considered: one is a $4.75 \%$ solution of $20 \%$ calibrated Intralipid ${ }^{27}$ and distilled water, filling a $25 \times 27$ $\times 19 \mathrm{~cm}$ black tank and giving a reduced scattering coefficient of about $10 \mathrm{~cm}^{-1}$. The second one consists of purified pork fat melted in a $19 \times 9 \times 12 \mathrm{~cm}$ tank and subsequently consolidated by cooling.

Time-resolved diffuse reflectance measurements were performed in the range 910-1080 nm. First, both samples are
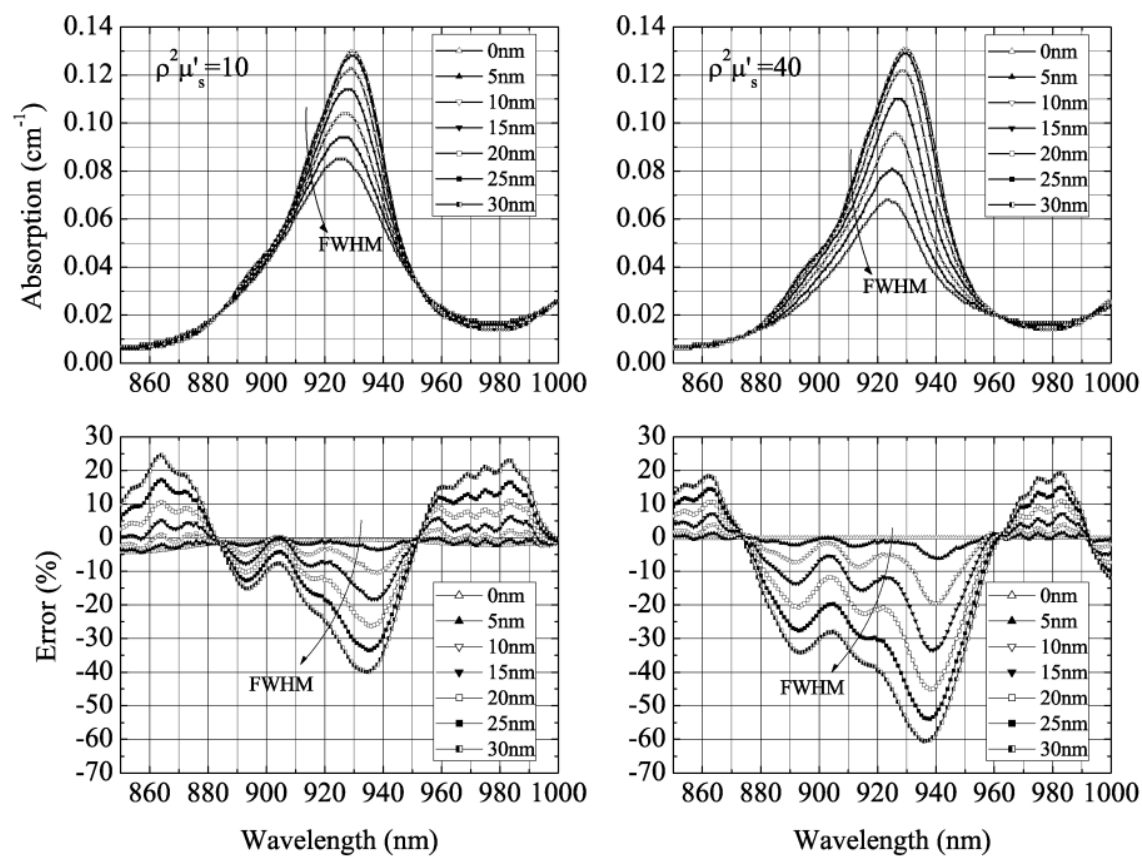

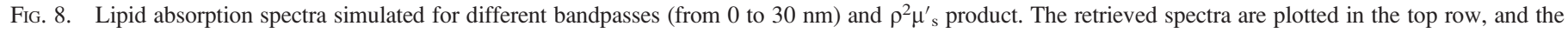
percentage errors are plotted in the bottom row. It can be seen that the absorption peak shifts toward lower wavelengths for increasing bandpasses. 


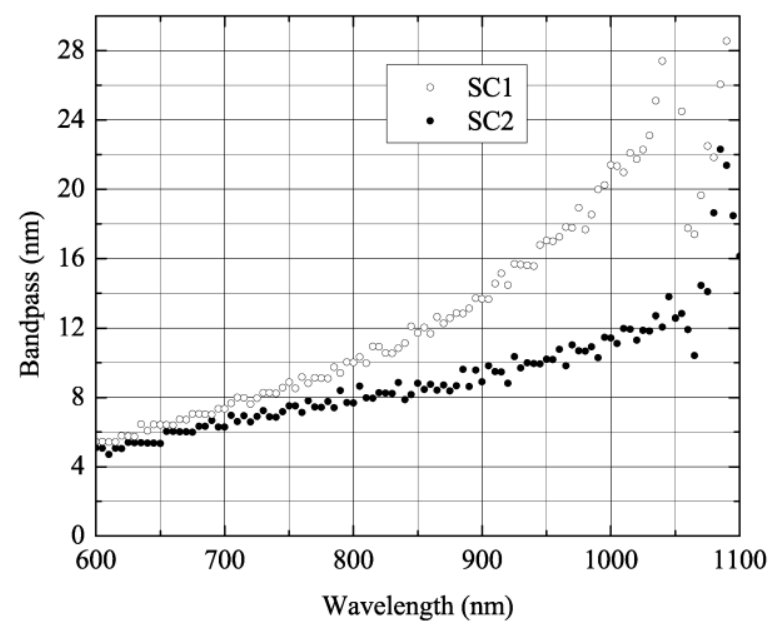

FIG. 9. Bandpass of the SC system in two different configurations.

measured with the TiS system at an inter-fiber distance of $2 \mathrm{~cm}$ in order to obtain a good signal-to-noise ratio without challenging the diffusion approximation. Subsequently, measurements are performed with the systems $\mathrm{SC} 1$ and $\mathrm{SC} 2$ at inter-fiber distances ranging from $1.5 \mathrm{~cm}$ to $4 \mathrm{~cm}$ depending on the available signal at the detector. For each wavelength, the absorption and reduced scattering coefficients were retrieved, using the same procedure described in the simulations section, by fitting the time-resolved curve to the diffusive time-resolved reflectance with the extrapolated boundary conditions for a semi-infinite medium. In order to take into account the nonideal instrumental response function (IRF), due to the finite duration of the laser pulse, finite response of the photomultiplier, and temporal dispersion in optical fibers, the theoretical curve was previously convoluted with the IRF.

The resulting absorption spectra are shown in Fig. 11 for the different setups and configurations at an inter-fiber distance of $2 \mathrm{~cm}$. The distortion of the calculated absorption is present in all cases. It is observed that this distortion is clearer for the lipid
TABLE I. Fitting results obtained by the spectrally constrained method applied to the lipid sample. The second column shows the inter-fiber distance, the third shows the concentrations retrieved assuming a monochromatic laser source, and the fourth shows the concentrations retrieved taking into account the real spectrum of the source.

\begin{tabular}{lccc}
\hline System & $\rho(\mathrm{cm})$ & $C_{1}$ & $C_{2}$ \\
\hline $\mathrm{SC} 1$ & 1.5 & 0.85 & 0.98 \\
& 2.0 & 0.82 & 1.01 \\
& 3.0 & 0.72 & 1.00 \\
$\mathrm{SC} 2$ & 4.0 & 0.69 & 0.98 \\
& 1.5 & 0.92 & 0.98 \\
& 2.0 & 0.91 & 0.98 \\
& 3.0 & 0.88 & 0.94 \\
& 4.0 & 0.87 & 0.94 \\
\hline
\end{tabular}

case than for the water case. This is clear from the fact that the lipid peak is narrower than the water peak. This also justifies the small difference between the water spectra retrieved by $\mathrm{SC} 1$ and SC2. In Fig. 12 the effect of the inter-fiber distance on the retrieved spectra is shown for the lipid case. The increasing of the inter-fiber distance generates a forward shift of the timeresolved curve, with a consequent photon path-lengthening inside the sample. The spectral distortions are therefore enhanced.

The distortion effect can be a strong drawback when Beer's law is applied to derive the chromophore concentrations in the sample. Here we propose a method that can be used in order to compensate this effect and improve the estimation of the concentrations. Since the absorption spectrum of a tissue is a linear combination of the chromophore spectra, it is possible to set the chromophore concentrations as unknowns in the fitting algorithm. The fitting procedure is carried out by minimizing the difference between the experimental and theoretical data of all time-resolved curves as a whole, by using a standard Levenberg-Marquardt algorithm. ${ }^{28}$ Starting from Eq. 3, theoretical curves at each wavelength are first generated by means of the source spectral shapes measured by an OMA and then convolved with the instrumental response function (IRF).

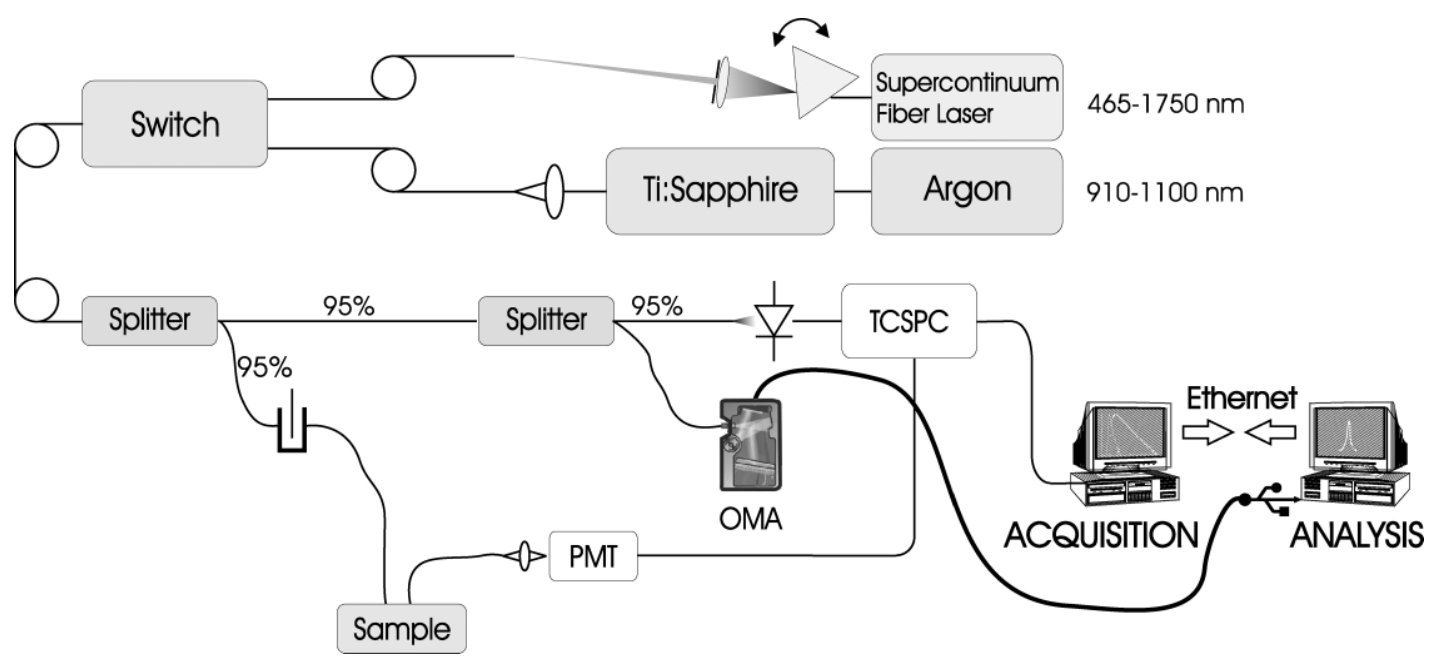

FIG. 10. Schematic of the experimental setup. The light coming from the supercontinuum source is dispersed by a rotating prism and coupled to a fiber by an

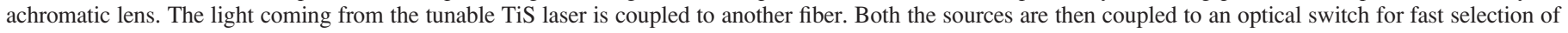

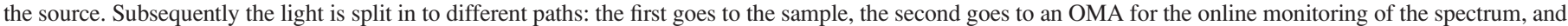

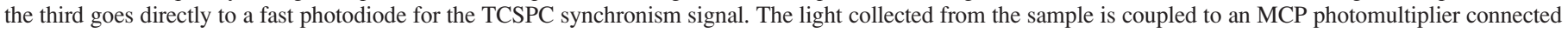
to a TCSPC board. 

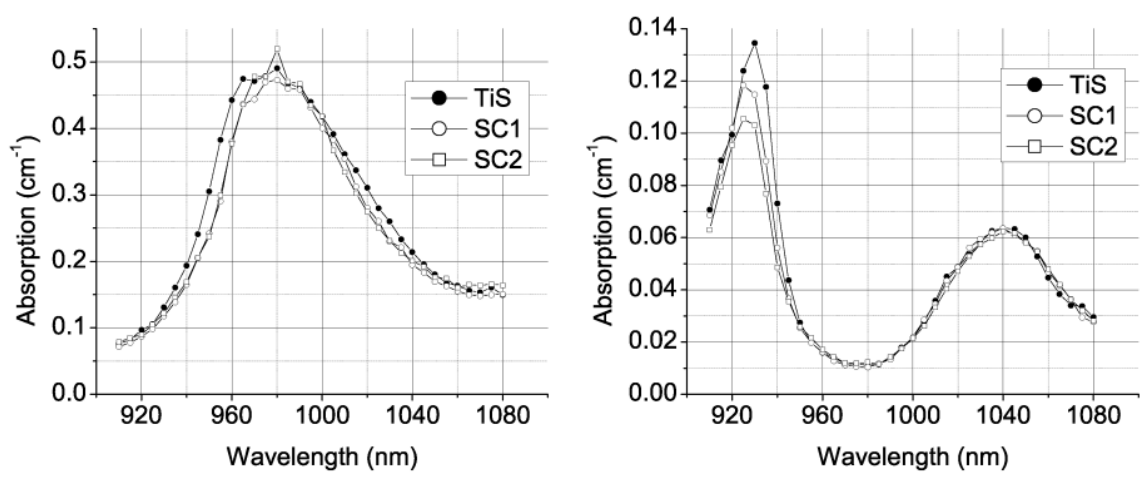

FIG. 11. (Left) Water and (right) lipid absorption spectra measured with the three systems at an inter-fiber distance of $2 \mathrm{~cm}$.

This method can only be applied if the chromophores present in the sample are known, as well as their absorption spectra. Table I shows the results of the fitting procedure for the lipid sample. The column labeled " $\mathrm{C}_{1}$ " shows the retrieved lipid concentration assuming a monochromatic system. The distortion manifests as an underestimation of the lipid concentration. In the column labeled " $\mathrm{C}_{2}$ ", results obtained with the real spectral response function of the system are presented. The retrieved concentrations are improved significantly: a maximum error of about $30 \%$ is obtained without the compensation, while the error reduces to a maximum of $6 \%$ by considering the bandpass of the instrument.

As outlined in the Introduction, photon migration is widely applied in different fields to estimate the absorption properties of highly diffusive media and then derive information on their composition for different purposes. Thus, a more accurate estimate of constituent concentrations can be relevant in several situations, such as the estimate of biological tissue composition for diagnostic purposes, including breast cancer detection and monitoring of the effectiveness of chemotherapy.

\section{CONCLUSION}

This work has investigated the effects of the broad bandpass of a time-resolved spectrophotometer for diffusive media. It has been shown that a distortion is introduced in the estimation of the absorption spectra due to cross-talk from spectral regions

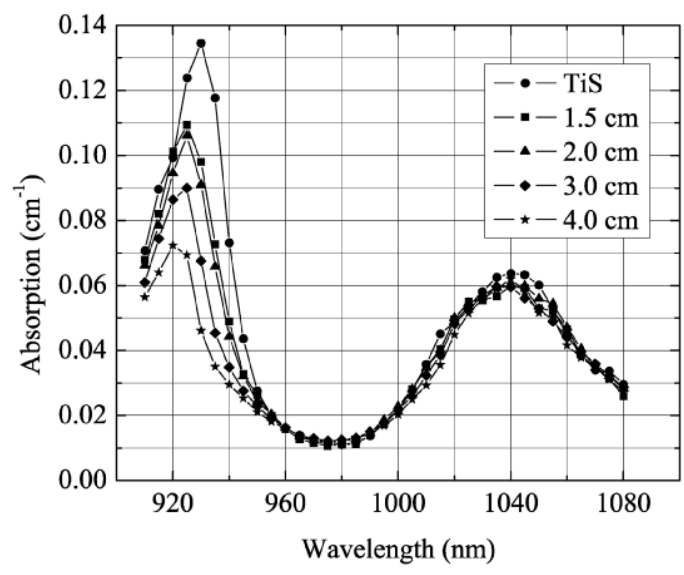

FIG. 12. Lipid absorption spectra retrieved by means of the SC2 system at different inter-fiber distances. The lipid spectrum retrieved by means of the TiS system at an inter-fiber distance of $2 \mathrm{~cm}$ is also shown for comparison. within the bandpass that exhibit different absorptions. In timeresolved photon migration the distortion is more severe as longer and longer photon TOFs are included in the data evaluation. It has also been shown that if the absorption spectra are not symmetric, the peak position can be shifted. Simulations and measurements have been performed on water and lipid in order to quantify this effect. Finally, a spectrally constrained method has been proposed for the assessment of the chromophore concentrations that is able to compensate for these distortions.

\section{ACKNOWLEDGMENTS}

The research leading to these results has received funding from the European Community's Seventh Framework Programme under the nEUROPt Project (grant agreement FP7-HEALTH-2007-201076).

1. S. Brodersen, J. Opt. Soc. Am. 43, 10 (1953).

2. S. Brodersen, J. Opt. Soc. Am. 44, 1 (1954).

3. C. L. Korb and C. Y. Weng, Appl. Opt. 43, 18 (2004).

4. H. J. Kostkowski and A. M. Bas, J. Opt. Soc. Am. 46, 12 (1956).

5. O. D. D. Soares and J. L. C. Costa, Appl. Opt. 38, 10 (1999).

6. A. Ishimaru, Wave Propagation and Scattering in Random Media (Academic, New York, 1978).

7. B. Tromberg, N. Shah, R. Lanning, A. Cerussi, J. Espinoza, T. Pham, L. Svaasand, and J. Butler, Neoplasia 2, 1 (2000).

8. G. Strangman, D. A. Boas, and J. P. Sutton, Biol. Psych. 52, 7 (2002).

9. E. E. Graves, J. Ripoll, R. Weissleder, and V. Ntziachristos, Med. Phys. 30, 5 (2003).

10. J. Johansson, S. Folestad, M. Josefson, A. Sparén, C. Abrahamsson, S. Andersson-Engels, and S. Svanberg, Appl. Spectrosc. 56, 6 (2002).

11. C. D'Andrea, A. Farina, D. Comelli, A. Pifferi, P. Taroni, G. Valentini, R. Cubeddu, L. Zoia, M. Orlandi, and A. Kienle, Appl. Spectrosc. 62, 5 (2008).

12. R. Cubeddu, C. D'Andrea, A. Pifferi, P. Taroni, A. Torricelli, G. Valentini, M. Ruiz-Altisent, C. Valero, C. Ortiz, C. Dover, and D. Johnson, Appl. Spectrosc. 55, 10 (2001).

13. A. Scheggi, S. Martellucci, A. Chester, and R. Pratesi, Biomedical Optical Instrumentation and Laser-Assisted Biotechnology (Kluwer Academic, Dordrecht, 1996).

14. J. K. Ranka, R. S. Windeler, and A. J. Stentz, Opt. Lett. 25, 1 (2000).

15. M. Rusu, A. B. Grudinin, and O. G. Okhotnikov, Opt. Exp. 15, 22 (2005).

16. S. Andersson-Engels, R. Berg, A. Persson, and S. Svanberg, Opt. Lett. 18, 20 (1993)

17. A. Bassi, J. Swartling, C. D’Andrea, A. Pifferi, A. Torricelli, and R. Cubeddu, Opt. Lett. 29, 20 (2004).

18. C. Abrahamsson, T. Svensson, S. Svanberg, S. Andersson-Engels, J. Johansson, and S. Folestad, Opt. Exp. 12, 17 (2004).

19. A. Pifferi, A. Torricelli, P. Taroni, D. Comelli, A. Bassi, and R. Cubeddu, Rev. Sci. Instrum. 78, 053103 (2007).

20. R. M. P. Doornbos, R. Lang, M. C. Aalders, F. W. Cross, and H. J. C. M. Sterenborg, Phys. Med. Biol. 44, 4 (1999).

21. F. Bevilacqua, A. J. Berger, A. E. Cerussi, D. Jakubowski, and B. Tromberg, Appl. Opt. 39, 34 (2000).

22. D. Contini, F. Martelli, and G. Zaccanti, Appl. Opt. 36, 19 (1997). 
23. W. H. Press, S. A. Teukolsky, W. T. Vetterling, and B. P. Flannery, Numerical Recipes in C: The Art of Scientific Computing (Cambridge University Press, New York, 1992).

24. R. C. Haskell, L. O. Svaasand, T.-T. Tsay, T.-C. Feng, M. S. McAdams, and B. J. Tromberg, J. Opt. Soc. Am. A 11, 10 (1994).

25. R. Cubeddu, M. Musolino, A. Pifferi, P. Taroni, and G. Valentini, IEEE J. Quantum Electron. 30, 10 (1994).
26. A. Bassi, A. Farina, C. D'Andrea, A. Pifferi, G. Valentini, and R. Cubeddu, Opt. Exp. 15, 22 (2007).

27. L. Spinelli, F. Martelli, A. Farina, A. Pifferi, A. Torricelli, R. Cubeddu, and G. Zaccanti, Opt. Exp. 15, 11 (2007).

28. C. D'Andrea, L. Spinelli, A. Bassi, A. Giusto, D. Contini, J. Swartling, A. Torricelli, and R. Cubeddu, Opt. Exp. 14, 5 (2006). 